\title{
Super-Resolution Image Reconstruction Based on Wavelet Transform and Edge-Directed Interpolation
}

\author{
Yingying Zhao and Aidi Wu \\ College of Science, Tianjin University of Technology and Education, Tianjin, 300222, China
}

\begin{abstract}
In this paper, a method of super-resolution image reconstruction based on wavelet transform and edge-directed interpolation is put forward. Firstly the low resolution image is decomposed by the stationary wavelet transform; meanwhile, the image obtained from edge-directed interpolation is decomposed by discrete wavelet transform, then the three high frequency sub bands obtained through stationary wavelet transform and discrete wavelet transforms are fused. The fused three high frequency sub bands are denoised. Finally the reconstructed high resolution image is gotten by invert wavelet transform. Experimental results show that this method can improve the quality of the reconstructed image with superior PSNR than the traditional methods.
\end{abstract}

Key words: Super-resolution image, wavelet transform, image fusion, threshold denoising.

\section{Introduction}

Super-resolution image reconstruction technique is to process low resolution images of single frame or multiple frames in order to obtain relatively high resolution images. This technique has been widely applied in the fields of video surveillance, seismic exploration, satellite remote sensing, medical imaging and image processing [1-4].

Image super-resolution technology can be divided into two classifications: frequency domain method and spatial domain method. The most common algorithm of super-resolution image reconstruction is method based on interpolation, such as nearest neighbor domain interpolation, bilinear interpolation, bicubic interpolation, etc. Interpolation algorithm has the advantage of simple intuition and lower computational complexity, but it doesn't produce an ideal effect, since essentially there is no increase of image information. Therefore, in this paper, we adopted the edge-directed interpolation, which can improve the edge blur and texture of the image when

Corresponding author: Yingying Zhao, master, research fields: wavelet analysis and signal processing. E-mail: 997868718@qq.com. compared with traditional interpolation methods. In addition, the interpolation algorithm is combined with other methods like wavelet transform [5]. A method

of super-resolution image reconstruction based on wavelet transform is put forward [6], Firstly perform wavelet transform on the low resolution images, make edge-directed interpolation in wavelet domain, and then use the wavelet inverse transformation to get the reconstructed super-resolution image, which shows more information including rich details. But the experimental results show that the super-resolution reconstructed images based on wavelet can have block effect, this is because of the wavelet coefficient is estimated according to blocks.

Compared with discrete wavelet, stationary wavelet transform has better stationary invariance advantage, so the reconstructed image is more approximate to the original images than discrete wavelet [7]. In image processing, four subband images obtained from wavelet transform have same scales. This stationary invariance can reprocess the wavelet coefficient much well and analyze the correlations between scales conveniently. This paper presents a new super-resolution reconstruction algorithm, which integrates edge-directed interpolation into 
super-resolution algorithm, fuse wavelet coefficients of stationary wavelet transform and discrete wavelet transform, and denoise each sub-image through threshold, finally get reconstructed high resolution image by inverse wavelet transform.

The remainder of this paper is organized as follows: In the Section 2, mathematical model of high resolution image is simply introduced; Edge-directed interpolation is discussed in the Section 3; In the Section 4, super-resolution reconstruction based on wavelet transform and edge-directed interpolation is studied; In Section 5, experimental results are discussed. And the conclusion is given at the end of this paper.

\section{Mathematical Model}

In the process of acquiring images, the motion blur is caused by the spread of light, the geometric deformation of an object in the process of movement and the insufficient sampling due to the influences of the hardware conditions can all lead to low image resolution. According to classical super-resolution reconstruction model, assume that the observed low resolution image can be described in the following equation:

$$
y=D H F x+n
$$

In this equation, $y$ is the observed low resolution image, $x$ is the unknown high resolution image, $F$ is geometric transform matrix, $H$ is motion blur matrix, $D$ is down sampling matrix, $n$ is random noise assumed to be Gaussion noise. The purpose of the super resolution reconstruction is to estimate the unknown high resolution image from the known low resolution images through Eq. (1).

Generally, due to the sparsity of drop mass matrix in reconstruction models, regularization method is usually used in solving Eq. (1). By adding regularization constraints to eliminate illposedness, the problem is translated into following an optimization problem.

$$
\hat{x}=\arg \min (\|D H F x-y\|+\lambda J(x))
$$

In this model, the first item is the data fidelity term which represents fitting degree between the images to be estimated and the real high resolution image; $J(x)$ is the regularization term providing proiri informations of the image, $\lambda$ is the regulation parameter which is used to balance the previous two terms.

\section{Edge-Directed Interpolation}

High resolution images obtained through traditional interpolation algorithm may blur the edge and the texture of image. In order to overcome these shortcomings, an interpolation algorithm preserving the edge, an edge-directed interpolation based on least-squares method is proposed, which improves the edge of the interpolation and obtains good results $[8,9]$.

The low resolution image is denoted as $G$ with size $m \times n$, and the reconstructed high resolution image is denoted as F with size $2 m \times 2 n$.

The first step: map the low resolution image to the grids of the high resolution image, let $\mathrm{F}_{2 i, 2 j}=G_{i, j}$, and interpolate in ldiagonal directions based on pixel points $F_{2 i, 2 j}$ to get $F_{2 i+1,2 j+1}$, as shown in Fig. 1,

That is

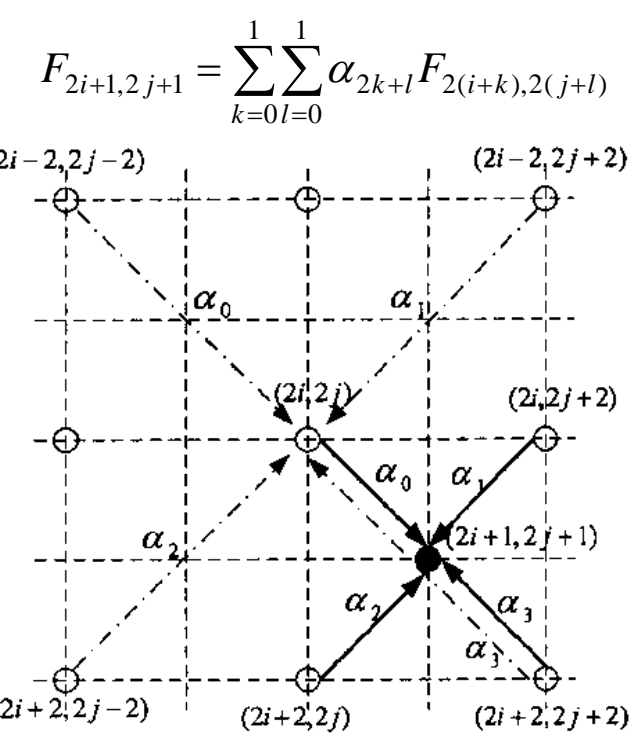

Fig. 1 Diagonal direction interpolation algorithm. 
In the above formula, $\alpha$ is the parameter, and we can get it by least square method through the relations between the known $F_{2 i, 2 j}$. Because there are similar local structures between low-resolution images and the high-resolution images, we have

$$
F_{2 i, 2 j}=\sum_{k=0}^{1} \sum_{l=0}^{1} \alpha_{2 k+l} F_{2(i+2 k-1), 2(j+2 l-1)}
$$

Rearranging $F_{2 i, 2 j}$ by lines to get column vector $\vec{y}=\left[y_{1} \ldots y_{k} \ldots y_{N^{2}}\right]^{T}$, above linear system of equations can be written as matrix form:

$$
\mathrm{C} \times \alpha=\vec{y}
$$

where, $C$ is a matrix of $N^{2} \times 4$.To solve this equation, use least square method to get the optimal solution, That is:

$$
\vec{\alpha}=\left(C^{T} C\right)^{-1}\left(C^{T} \vec{y}\right)
$$

Then get $\mathrm{F}_{i, j}(i+j=$ even $)$

The second step: to get $\mathrm{F}_{i, j}(i+j=$ odd $)$, we use cross direction interpolation as shown in Fig. 2.

Similarly, $\quad \mathrm{F}_{i, j}(i+j=$ odd $)$ can be obtained through Cross direction interpolation and least square method Based on $\mathrm{F}_{i, j}(i+j=e v e n)$, thus an high resolution image based on Edge-directed interpolation is obtained.

Edge-directed interpolation algorithm overcomes the deficiency of the traditional interpolation algorithm to a certain extent, and improves the image block effect and the image edge blurr. The image obtaind through this method is improved significantly.

\section{Super-Resolution Reconstruction Based on Wavelet Transform and Edge-Directed Interpolation}

Combining the advantages of edge-directed interpolation and the wavelet transform, a super-resolution image reconstruction algorithm based on wavelet transform and edge-directed interpolation is put forward. Edge-directed interpolation algorithm, superior to traditional interpolation algorithms, improves the edge blurr effect and the sawtooth effect. Integrate the high frequency sub image in three directions (horizontal, vertical and diagonal direction) generated respectively by discrete wavelet and stationary wavelet transforms [10]. Noise of the fused high frequency sub image is suppressed by threshold method. Finally taking the original low resolution image as low frequency sub image, the reconstructed high resolution image is gotten through invert wavelet transform. This process is as shown in Fig. 3.

The reconstruction steps of high resolution image based on wavelet transform and edge-directed interpolation are as follows:

(1) input low resolution image $y$.

(2) Decompose y by the stationary wavelet transform,

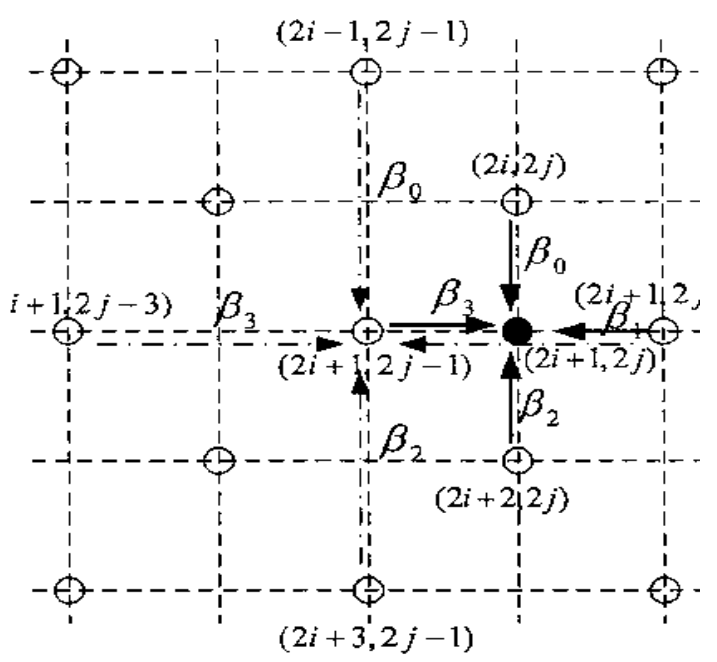

Fig. 2 Cross direction interpolation algorithm.

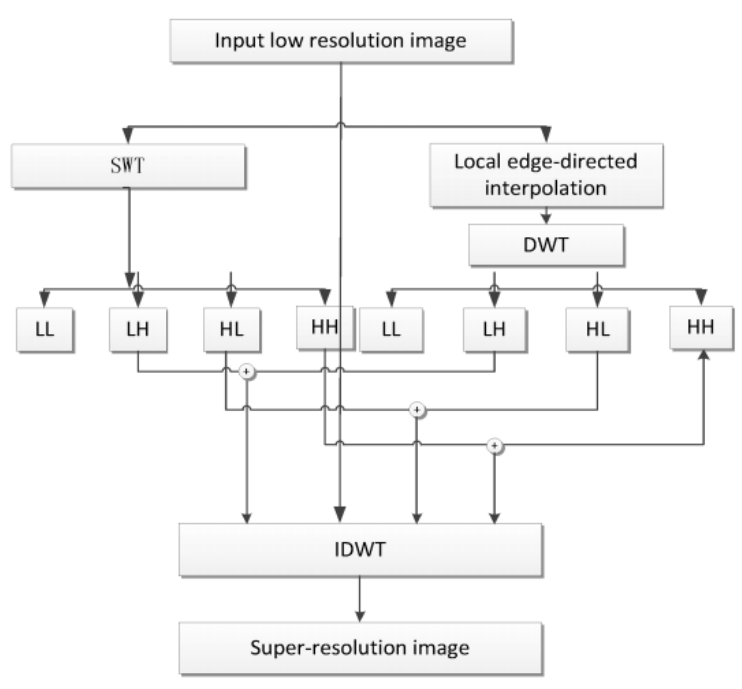

Fig. 3 High resolution image algorithm based on wavelet transform and edge-directed interpolation. 


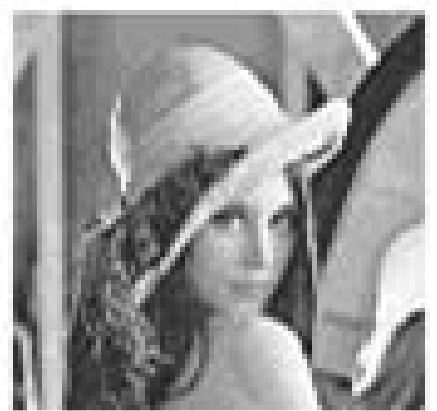

(a) low-resolution image

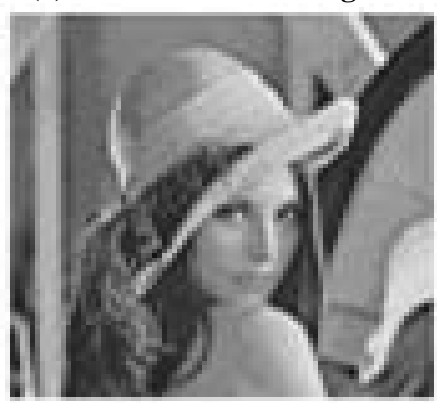

(c) edge-directed interpolation

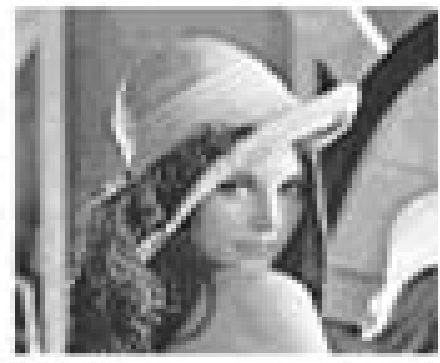

(e) original image

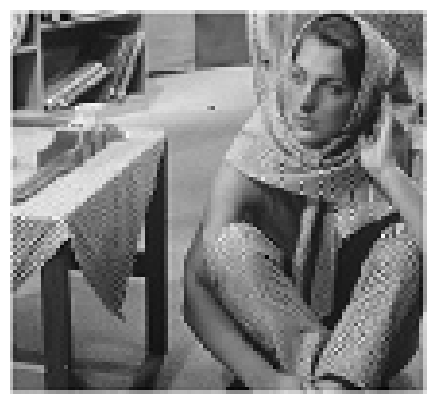

(a) low-resolution Image

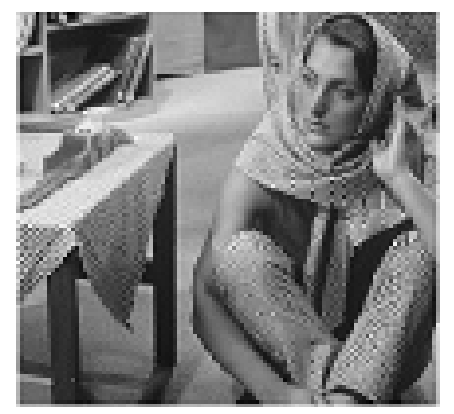

(c)the edge-directed

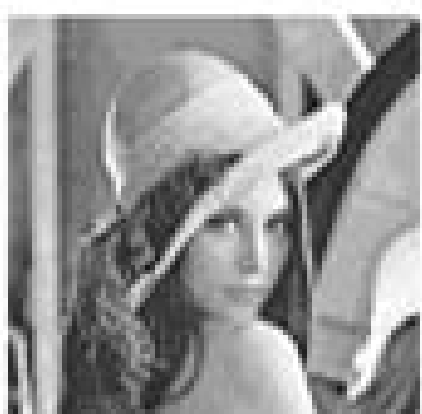

(b) linear interpolation method

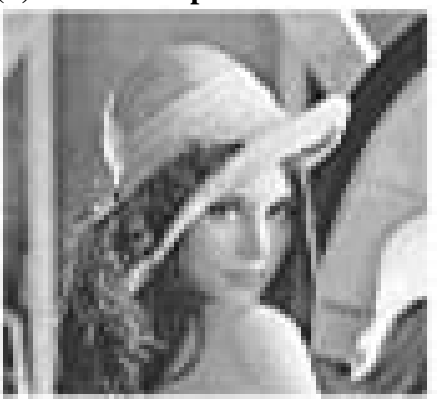

(d) wavelet method

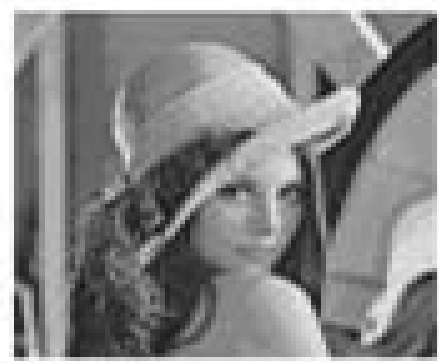

(f) The proposed method

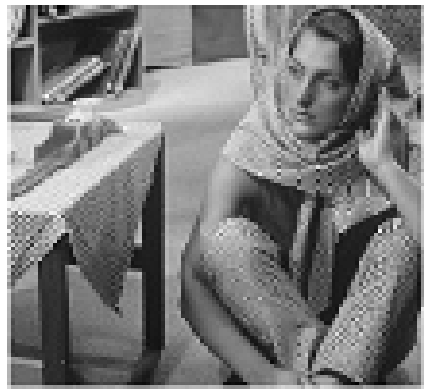

(b)The linear interpolation method

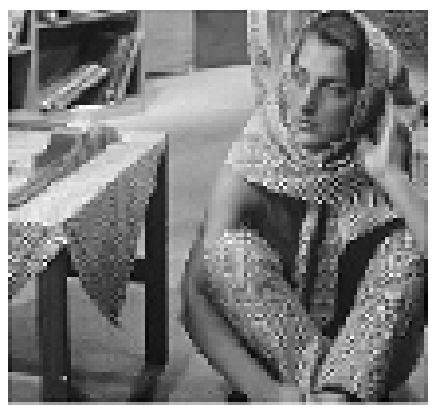

(d)The wavelet method interpolation 


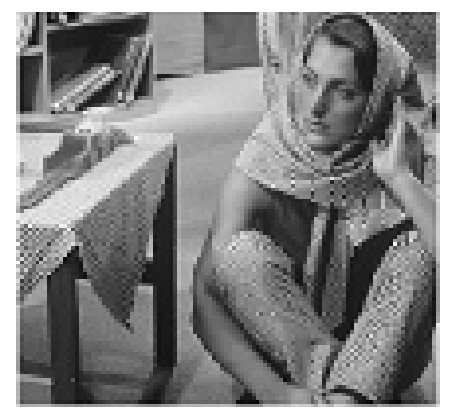

(e) original image

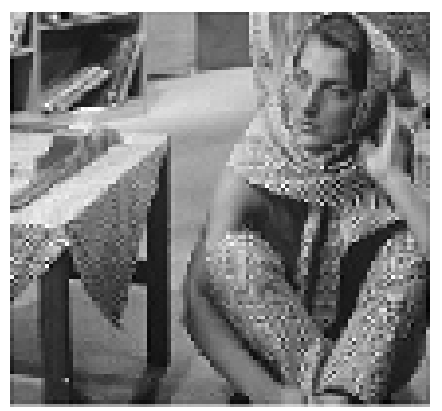

(f) The proposed method

Fig. 4 Comparison among the reconstructed image method.

Table 1 PSNR of the reconstructed images.

\begin{tabular}{lllll}
\hline Image & Linear interpolation & Edge-directed interpolation & Wavelet method & Proposed method \\
\hline Lena & 26.5276 & 26.8197 & 24.1 & 26.9273 \\
Woman & 22.2 & 22.9 & 20.2 & 23.1 \\
\hline
\end{tabular}

get three high frequency sub images $y h 1, y c 1, y d 1$ (horizontal, vertical and diagonal direction).

(3) Perform edge-directed interpolation on y, and interpolated image is decomposed by discrete wavelet transform to get three high frequency sub images $y h 2, y c 2, y d 2$ (horizontal, vertical and diagonal direction).

(4) Fuse the three high frequency sub images obtained from step (2) and step (3):

$$
\begin{aligned}
& y h=t \times y h 1+(1-t) y h 2 \\
& y c=t \times y c 1+(1-t) y c 2 ; \\
& y d=t \times y d 1+(1-t) y d 2
\end{aligned}
$$

(5)Threshold de-nosing, take threshold function as

$$
d_{m}(y)=s_{T}(y)=\left\{\begin{array}{cc}
y-T, & y>T \\
y+T, & y<-T \\
0, & |y| \leq T
\end{array}\right.
$$

(6) Get the reconstructed high resolution image $x$ through invert wavelet transform.

\section{Experimental results}

We take PSNR as the quality index of reconstruction. The higher PSNR is, the better quality of the reconstructed image is. PSNR can be defined as

$$
P S N R=10 \log _{10} \frac{255^{2}}{M S E}
$$

In this formula

$$
M S E=\frac{1}{N_{1} N_{2}} \sum_{i=1}^{N_{1}} \sum_{j=1}^{N_{2}}[f(i, j)-\hat{f}(i, j)]^{2}
$$

represents mean square error. $f(x, y)$ and $\hat{f}(x, y)$ represent the original image and reconstructed image respectively. $N_{1}$ and $N_{2}$ are the dimensionalities of the image.

In order to verify the performance of the algorithm, we make simulated test with Matlab software. Lena image with size $256 \times 256$ is chosen as test image. Firstly take smooth filer and down samplings to get low resolution image with size $128 \times 128$, and then use linear interpolation, edge-directed interpolation, wavelet transform and the method proposed in this paper to reconstruct high resolution images. Results are shown in Fig. 4 and Table 1. We can see from the data in Table 1 that the method proposed in this paper provides higher PSNR and better image edge information than the other three ones.

\section{Conclusion}

In this paper, a method of super-resolution image reconstruction based on wavelet transform and edge-directed interpolation is put forward, which effectively keeps the image edge details by making full use of the advantages of stationary wavelet transform and edge-directed interpolation. It is superior to traditional algorithms from both subjective visual effect and objective measurement 
standards.

\section{References}

[1] Milanfar, P. 2010. Super-resolution Imaging. USA: CRC Press.

[2] Park, S. C., Park, M. K., and Kang, M. G. 2003. "Super-Resolution Image Reconstruction: A Technical Overview.” IEEE Signal Processing Magazine 20: 21-36.

[3] Moon, G. K., and Chaudhuri, S. 2003. "Super-Resolution Image Reconstruction.” IEEE Signal Processing Magazine 20: 19-20.

[4] Bose, N. K., Lertrattanapanich, S., and Chappalli, M. B. 2004. "Super-Resolution with Second Generation Wavelets.” Signal Processing: Image Communication 19 (May): 387-91.

[5] Bose, N. K., Lertrattanapanich, S., and Chappalli, M. B. 2004. "Super-Resolution with Second Generation
Wavelets.” Signal Process: Image Commun 19: 387-91.

[6] Hong, J. T., Xin, J. T., and Jian, L. 2003. "Super-Resolution Remote Sensing Image Processing Algorithm Based on Wavelet Transform and Interpolation.” In Proceedings of SPIE, 259-63.

[7] Hasan, D., and Gholamreza, A. 2011. "Image Resolution Enhancement by Using Discrete and Stationary Wavelet Decomposition.” Image Proceedings 20: 1458-60.

[8] Li, X., and Orchard, M. T. 2001. "Edge-Directed Prediction for Lossless Compression of Natural Image.” IEEE Transactions on Image Proceeding 10: 813-17

[9] Li, X., and Orchard, M. T. 2001. "New Edge-directed interpolation.” IEEE Transactions on Image Proceedings 10: 1521-72.

[10] EL-KHAMY S E, HADHOUD M M,DESSOUKY M I, et al. A new super-resolution image reconstruction algorithm-based on wavelet fusion[C]// Proceedings of the 22nd National Radio Science Conference. Piscataway. IEEE, 2005:195-204. 